\section{Matter all in the mind}

\section{Kurt Gottfried}

n the autumn of 1925, Paul Adrien Maurice

Dirac, a Cambridge University graduate

student of very few words, astonished the world of physics with a remarkable paper on quantum mechanics. His publication heralded the arrival of a mind of exceptional originality and power at the frontier of physics.

Werner Heisenberg's discovery of quantum mechanics sprang from the fertile soil of Göttingen and Copenhagen, not Cambridge. Nevertheless, Dirac, knowing only Heisenberg's ground-breaking but rather mysterious and fragmentary paper, produced an almost complete formulation of quantum mechanics wholly on his own, mirroring the achievement of the experienced Göttingen collaboration of Max Born, Pascual Jordan and Heisenberg. Born recalled this as "one of the greatest surprises of my scientific life, for the name of Dirac was completely unknown to me".

Dirac, born 100 years ago this year, often displayed an uncanny ability, whenever the need arose, to invent deep mathematical concepts that were new to physicists. Readers of his early paper would ask by what magic had he turned his austere, abstract constructs into quantitative descriptions of physical phenomena. Dirac's genius was quickly recognized - he was the youngest participant in the élite Solvay Congress of 1927, at which Niels Bohr and Albert Einstein began their long debate about the foundations of quantum mechanics. On first meeting him there, the formidable Wolfgang Pauli quipped that Dirac believed "there is no God, and Dirac is His prophet".

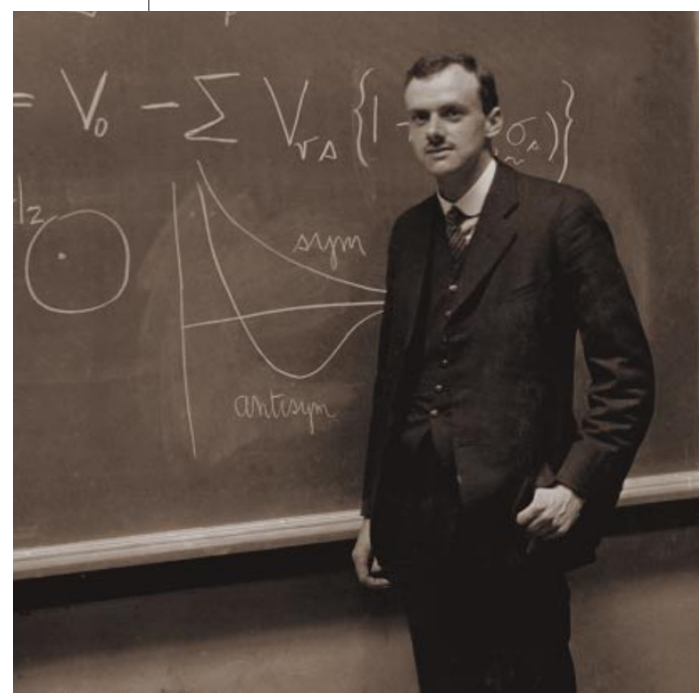

Truth is beauty: Paul Dirac and the quantum mechanics of the hydrogen molecule.
The development of nonrelativistic quantum mechanics - the microscopic counterpart of newtonian mechanics - was essentially complete by the end of 1926. But it was not known how to extend the new theory to the electromagnetic field, or to particles moving with velocities approaching the speed of light. Dirac solved both problems, giving birth to quantum electrodynamics.

Although Dirac's 'quantization' of classical electrodynamics yielded no great surprise, it was the first consistent and correct account of the absorption, emission and scattering of light. Ultimately, all the optical phenomena with which we are so familiar are fully described by Dirac's radiation theory of 1927.

By contrast, Dirac's relativistic wave equation for electrically charged particles, developed the following year, held many surprises - as he later put it, the equation is much more intelligent than its inventor. However one interprets this remark, the invention itself is an unsurpassed example of a disciplined imagination obtaining profound insights into the natural world.

Dirac's equation, when applied to the electron, describes accurately its magnetic moment, and elucidates fine details in the spectrum of hydrogen that are not accounted for by the nonrelativistic theory. Yet the equation seemed to have a terrible flaw, because it also had solutions with negative energy that made no apparent sense. Much effort was devoted to eliminating them, and when that failed, to understanding their significance.

After various inconsistent attempts by himself and others, Dirac made the spectacular proposal that these solutions describe a hitherto unknown particle that has the same mass but the opposite charge to the electron. Furthermore, he predicted that when this 'positron' encounters an electron, the pair would annihilate into two or more photons, and, conversely, that electron-positron pairs could be created by the interaction of energetic photons with matter. Soon after, the positron was discovered in cosmic-ray experiments, and was eventually found to have all the properties predicted by Dirac's theory.

Physics has produced other far-fetched predictions that have subsequently been confirmed by experiment. But Dirac's prediction of antimatter stands alone in being motivated solely by faith in pure theory, without any hint whatever from data, and yet revealing a deep and universal property of nature. The positron illustrates the general principle that all particles that have a conserved attribute, such as charge, have corresponding antiparticles. Furthermore, the existence of antimatter was soon understood to be an inevitable consequence of a

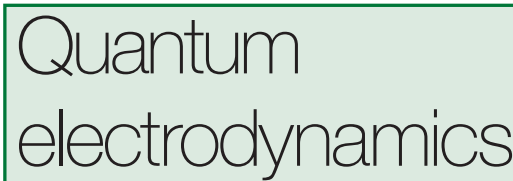

Paul Dirac's prediction of antimatter stands alone in being motivated solely by faith in pure theory, without any hint whatever from data.

consistent marriage of relativity with quantum mechanics. A closely related lesson was that the particles that emerge from a reaction need not have existed beforehand - they can be created in the process itself, by the transformation of energy into matter. This fact has become so well established that it is now treated as mundane, but at the outset Dirac's theory was dismissed as incredible by Bohr and other leading figures, even as the evidence for the positron was accumulating.

Quantum electrodynamics has survived more searching experimental scrutiny than any other physical theory. It is valid down to distances more than six orders of magnitude shorter than those that had been explored at the time Dirac invented it. This accomplishment relies on great refinements in mathematical techniques, some of which, such as Richard Feynman's path integral, stem from ideas originating with Dirac. Today, quantum field theory, of which quantum electrodynamics is the prototypical core, is universally considered to be the most successful, fruitful description of the basic constituents of matter and their interactions.

Dirac's creations are a constant presence in today's physics. His abstract, elegant style is now the standard language of quantum mechanics. His maxim that "physical laws should have mathematical beauty" guides throngs of physicists who pursue highly speculative research in the hope that they, too, can discover deep truths his way. The future may confirm another of Dirac's remarkable and brilliant ideas - that magnetic monopoles should exist, as this would explain why all particles carry an electrical charge of an integer multiple of the electronic charge. Indeed, Dirac monopoles, and closely related constructs, loom large in attempts to forge a unified theory of the fundamental interactions.

Kurt Gottfried is in the Laboratory of Nuclear Studies, Cornell University, Ithaca, New York 14853, USA.

\section{FURTHER READING}

Schweber, S. S. QED and the Men Who Made It (Princeton Univ. Press, 1994).

Kragh, H. Dirac: A Scientific Biography (Cambridge Univ. Press, 1990).

Miller, A. I. Early Quantum Electrodynamics

(Cambridge Univ. Press, 1994). 